assessment of these two parameters after hospital admission. Finally, the incidence of on-site intubation and following arrival at the hospital, and the respective times of arrival of the severely and not severely injured at a hospital were compared.

Results: A total of 245 persons were injured. The average age of those injured was 17.3 years. The median of the TBSA of all of the injured was $12 \%$ and 87 patients (36\%) had sustained an inhalation injury. Agreement between onsite $(n=46)$ and final TBSA assessment was poor (PCC $=$ $\left.0.77 ; R^{2}=0.60\right)$. The estimates of total body surface area burned at the $\operatorname{ED}(n=78)$ were more accurate than were those obtained prior to transport to the $\mathrm{ED}(\mathrm{PCC}=0.96$; $\mathrm{R}^{2}=0.93$ ). The diagnosis of inhalation injury, both on-site ( $n=79$, sensitivity $=100 \%$, specificity $=24 \%)$ and in the $\operatorname{ED}(n=198$, sensitivity $=99 \%$, specificity $=36 \%$ ), was sensitive, but not specific. Eight patients were endotracheally intubated on-site. Their injury and trauma parameters did not differ from those of the 73 patients who were intubated in the hospital. Also, there was no substantially significant difference in the time of arrival at a hospital between the severely and non-severely injured $(p=0.55)$.

Conclusions: In this major burn accident, the TBSA burned was not estimated reliably in the non-clinical environment. The diagnosis of inhalation injury was adequate, but resulted in over-triage on-site and at the ED. On-site triage did not lead to priority transport for the severely wounded. Detailed assessment of injuries of burn casualties only is practical in a specialized clinical setting. The early transfer of victims to a hospital, therefore, has the greatest priority in major incidents involving burn casualties. Keywords: burns; fire; Netherlands; resuscitation; triage Prebosp Disast Med 2005:20(3):s135-s136

\section{Hospital Disaster Planning-A Need-Based Phased Response}

T.J. Ligthelm

South Africa National Defence Force, South Africa

Healthcare facilities are a very expensive commodity to activate to a state of "standby" in a possible, yet unconfirmed, external disaster situation. Irrespective of any arrival of patients, the preparations are disruptive to hospital routine, which cost money, and disposables opened in preparation are often wasted. Therefore, it is essential to activate only the required resources and hospitals, in a reliable approach. In a country where the health service consists of a combination of public, military, and private hospitals, an even more accurate coordination is required for effective disaster relief. Effective coordination structures are needed to address this unique multi-service approach to effective hospital disaster response.

A simple planning model for hospital disaster planning, consisting of appreciation, planning, implementation, and evaluation processes, will provide the basic requirements for a phased response. Based on emergency service capabilities and the nature of the disaster, the primary responding hospitals within a geographical area are identified. Based on a previously agreed-upon flowchart, secondary and ter- tiary responding hospitals are activated to ensure the most economical use of resources and to address the influx of patients.

Hospital disaster planning is never an absolute science, but by using specific indicators as well as accepting unpredictable variables, optimum utilization of resources will be possible to address an external disaster.

Keywords: disaster; hospital; indicators; planning; resources, use of Prebosp Disast Med 2005;20(3):s136

\section{Utilization of Strong Authentication in Disaster Situations}

A. Immonen; M.A.K. Mattila; J. Holopainen

University of Kuopio, Finland

Introduction: One of the major problems in disaster situations is that it is difficult to determine the number, identity, condition, and location of casualties, injured, and rescued persons. This results in unnecessary mental distress and a waste of professional work. In order to avoid and diminish this drawback, an effective application to document all people in the disaster area must be created. Because documentation technology is already used effectively in other areas, there is no reason not to adapt analogous technology into disaster management.

Methods: Strong authentication methods, i.e., smart cards, token devices, or bio signals, combined with radio frequency identification (RFID) are practical methods to identify people and follow their whereabouts. Once booked into a centralized database, information can be utilized for many actual purposes.

Discussion: Individually created smart cards or tokens would include personal data, i.e., date of birth, medication, possible traumas, professional skills, photograph, and fingerprints. All information in the mobile network should be in real-time and readable in command centers, field hospitals, and in areas where food, water, medication, clothing, and accommodation supplies are distributed. Also, mobile detectors could be used.

In humanitarian aid, all people should be treated equally. Without mechanisms to identify people and document their actions, there is always a possibility of displacement and fraud. Real-time situation and location reports allow medical staff to develop the logistics more effectively than in the present situation.

Conclusion: No solutions of this type have been tested or evaluated in real emergencies. All essential equipment, smart identification cards, readers/writers, detectors, and mobile wireless networks are commercially available, but first need to be tested in disaster drills and then in minor scale incidences. Because technology is extremely difficult to implement in sudden and strange conditions, it is recommended that international rescue groups are prepared to construct a type of "ad hoc" identification network.

Keywords: disasters; documentation; identification; management; network; triage

Prebosp Disast Med 2005;20(3):s136 